\section{Endoscopic ultrasound-assisted transpapillary stent placement}

Endoscopic retrograde pancreatography (ERCP) has become a standard approach to the treatment and evaluation of chronic pancreatitis $[1,2]$. When anatomical variations and previous surgical interventions preclude the use of ERCP, antegrade cannulation of the pancreatic duct using endoscopic ultrasound (EUS) can be performed $[3,4]$. We present a case of EUSassisted antegrade cannulation of the pancreatic duct which facilitated the placement of a transpapillary stent.

A 57-year-old man was referred for evaluation of obscure, gastrointestinal bleeding associated with acute, recurrent pancreatitis. Colonoscopy and upper endoscopy were unremarkable. EUS examination revealed evidence for chronic pancreatitis. Also visualized by EUS was a pseudoaneurysm of the gastroduodenal artery which was compressing the ventral pancreatic duct. The patient was referred for interventional radiology and the pseudoaneurysm was embolized using coils.

Two months later, the patient showed no further signs of gastrointestinal bleeding. However, he continued to have episodes of acute pancreatitis. ERCP revealed a stricture of the ventral pancreatic duct with proximal ductal dilatation (Figure $1 \mathbf{a}$ ). Despite multiple attempts, deep cannulation of the pancreatic duct failed.

Subsequently, a linear EUS scope was introduced into the stomach and a 19-gauge Echotip needle (Wilson-Cook, WinstonSalem, North Carolina, USA) was inserted through the stomach and into the pancreatic duct. A 0.035" Jagwire (Boston Scientific, Natick, Massachusetts, USA) was deployed antegradely into the pancreatic duct, past the ampulla, and into the duodenum (Figure $\mathbf{1 b}$ ). The EUS scope and needle were then removed, leaving the wire in place. The wire was then snared in a rendezvous fashion by duodenoscopy (Figure 2). Using the wire in place, a $5 \mathrm{Fr}$ by $7 \mathrm{~cm}$ pancreatic stent was deployed retrogradely across the ampulla. The patient was discharged home, with no fur-
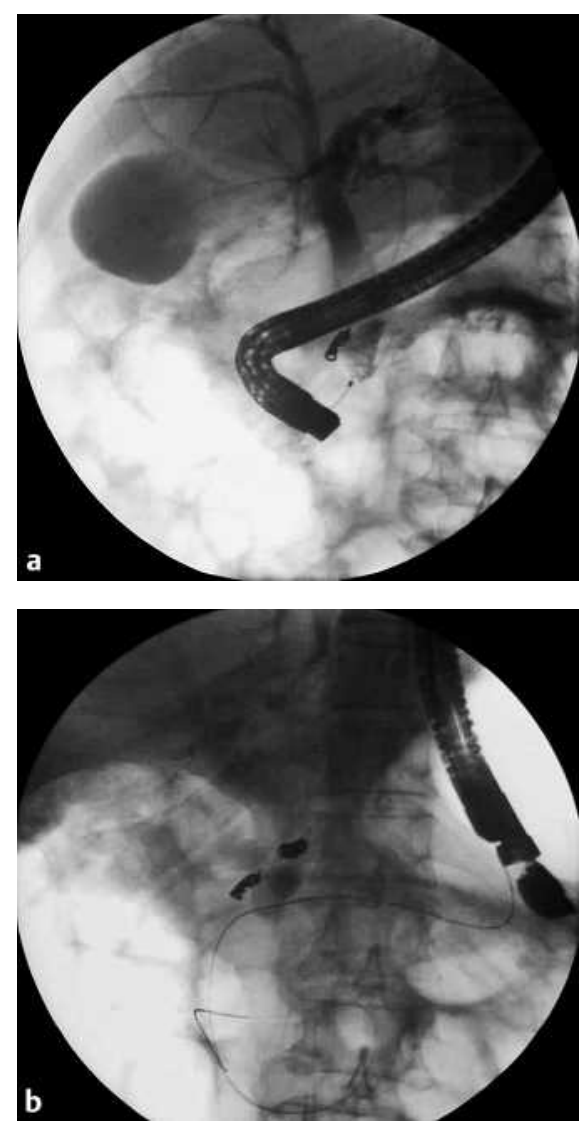

Figure 1 Fluoroscopic images of the pancreatic duct. a Endoscopic retrograde cholangiopancreatography (ERCP) reveals a distal stricture of the pancreatic duct with mild ductal dilatation proximally. Coils from prior embolization can clearly be seen. b An endoscopic ultrasound (EUS) scope is shown with a wire passing antegradely, through the pancreatic duct, and into the duodenum.

ther episodes of pancreatitis or bleeding having occurred up to 1 year later.

In this case, successful placement of a transpapillary stent using EUS and a rendezvous technique was performed, resulting in a good clinical outcome. To our knowledge, this is the first report of transpapillary stent placement via this approach.

Endoscopy_UCTN_Code_TTT_1AS_2AD

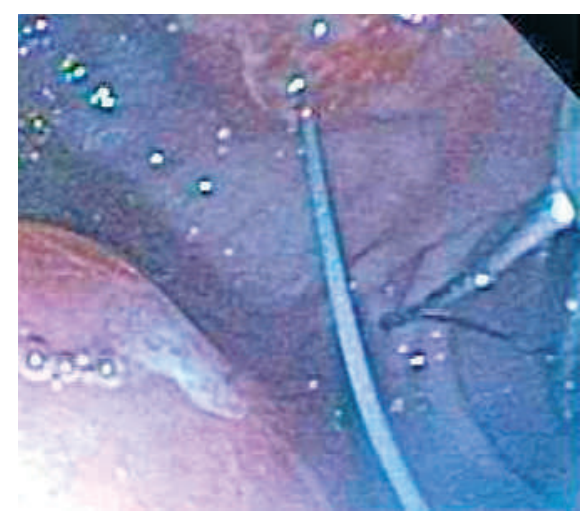

Figure 2 An endoscopic view from the second portion of the duodenum. A wire is seen coming out of the papilla, and a snare is ready

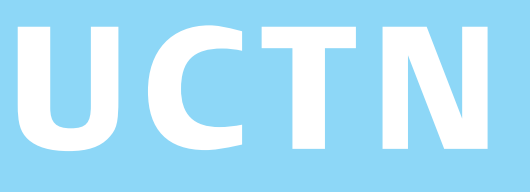

to retrieve the wire in a rendezvous fashion.

\section{R. D. Anderson, D. Mallat}

Baylor University Medical Center, Dallas, Texas, USA.

\section{References}

${ }^{1}$ Lehman G. Role of ERCP and other endoscopic modalities in chronic pancreatitis. Gastrointest Endosc 2002; 56 (6 Suppl): S237-S240

${ }^{2}$ Neuhaus H. Therapeutic pancreatic endoscopy. Endoscopy 2004; 36: 8-16

${ }^{3}$ Kahaleh, M, Yoshida C, Yeaton P. EUS antegrade pancreatography with gastropancreatic duct stent placement: review of two cases. Gastrointest Endosc 2003; 58: $919-$ 923

${ }^{4}$ François E, Kahaleh M, Giovannini M et al. EUS-guided pancreaticogastrostomy. Gas-

\section{Corresponding Author}

\section{R. D. Anderson}

3rd Floor, Truett Bldg. 3500 Gaston Avenue Dallas, TX 75246

USA

Fax: $\quad+1-214-820-8168$

E-mail: bobandersmd@yahoo.com trointest Endosc 2002; 56: 128 - 133 